\title{
群 $Z_{2}$ 在球面 $S^{n}$ 上的光滑作用 ${ }^{*}$ \\ 杨 华 建 \\ (华南师范大学数学系, 广州 510631)
}

关评调维球从、Gysin 叙列、对合㑊边

\section{一、引言及主要结果}

讨论和决定一个给定光滑闭流形上的所有可能光滑对合的协边分类, 是对合流形理论研 究中一项很有意义的工作. 但一直缺乏有效方法. 目前除文献 1 1]外尚未有其它结果. 利用 一类结构群为 $Z_{2}$ 的纤维丛流形, 本文将建立一种分类方法. 作为这种方法的应用, 我们将 决定 $S^{*}$ 上光滑对合的协边分类.

约定以下出现的流形和对合都是光滑的, 所有协边关系都不定向. 记闭流形 $M^{*}$ 的协边 类为 $\left[M^{n}\right]_{2}, M^{n}$ 上的对合 $\tau$ 为 $\left(M^{n}, \tau\right),\left(S^{m}, A\right)$ 为 $S^{m}$ 上的对径对合, $\left(S^{m}, A_{0}\right)$ 为 $S^{m}$ 上的对合, $A_{0}\left(x_{0}, x_{1}, \cdots, x_{m}\right)=\left(-x_{0}, x_{1}, \cdots, x_{m}\right)$.

对于给定的对合 $\left(M^{n}, \tau\right), A \times \tau$ 为 $S^{m} \times M^{*}$ 上的自由对合, 粘合 $S^{m} \times M^{*}$ 中所有点 对 $(x, y)$ 与 $(A x, \tau y)$, 即可得一个 $n+m$ 维流形 $R^{m}(\tau)=S^{m} \times M^{n} / A \times \tau$. 现设 $p: S^{m} \rightarrow$ $R P(m)$ 为投射, 粘合 $S^{m}$ 中所有点对 $x$ 与 $A x$, 则易知 $\left(S^{m}, p, R P(m)\right)$ 为主 $Z_{2}$ 丛, 由文献 [2] 定义 5.1, $R^{m}(\tau)$ 为 $R P(m)$ 上以 $M^{\prime \prime}$ 为纤维、结构群为 $Z_{2}$ 的纤维丛. 我们将证明

定理 1 两个对合 $\left(M_{1}^{*}, \tau_{1}\right),\left(M_{2}^{n}, \tau_{2}\right)$ 协边当且仅当流形 $R^{m}\left(\tau_{1}\right)$ 协边于流形 $R^{m}\left(\tau_{2}\right)$ 对一切 $m \geqslant 0$. 故对合 $\left(M^{*}, \tau\right)$ 协边类为 0 当且仅当流形 $R^{m}(\tau)$ 的协边类为 0 对一切 $m \geqslant 0$.

定理 1 建立了用纤维丛流形 $R^{m}(\tau)$ 分类对合的一种方法. 特别地, 当 $\left(M^{*}, \tau\right)$ 为 $\left(S^{*}, \tau\right)$ 时, $R^{\prime \prime}(\tau)$ 总为纤维球丛, Gysin 叙列使得我们能够证明

定理 2 任对合 $\left(S^{*}, \tau\right), R^{m}(\tau)$ 的协边类总为 0 对一切 $m \geqslant 0$. 因而 $\left(S^{n}, \tau\right)$ 总具 0 协边类, $\tau$ 总可扩张为某带边为 $S^{n}$ 的 $n+1$ 维流形上的对合.

对任何对合 $\left(M^{*}, \tau\right)$, 记其不动点集 $F$ 在 $M^{*}$ 中的法纵为

$$
\nu \rightarrow F=\bigcup_{k}\left(\nu^{n-k} \rightarrow F^{k}\right) \text { (不交并), }
$$

这里 $F^{k}$ 为 $F$ 中全部 $k$ 维子流形的不交并. 对于 $\left(S^{n}, \tau\right)$, 由 $\left(S^{n}, \tau\right)$ 协边类为 0 可得 $\nu \rightarrow F$ 作为向量丛具有 0 协边类. 从而得到

本文 1990 年 4 月 4 日收到. 1990 年 7 月 2 日收到修改稿.

*国家自然科学基金资助项目. 
定理 3 任何 $\left(S^{*}, \tau\right)$ 的不动点集或者是空集, 或者每个 $F^{k}$ 都为某个 $k+1$ 维流形的 边界. 特别地, 孤立不动点必为偶数个.

到此， $S^{*}$ 上对合的协边分类问题获得解决.

\section{二、定理的证明}

引理 1 设对合 $\left(M^{*}, \tau\right)$ 不动点集法丛为 $\nu \rightarrow F$, 则流形 $R^{m}(\tau)$ 协边于实射影空间 丛流形 $R P(\nu \oplus(m+1) R)$.

证设 $I$ 为 $M^{*}$ 上的恒同对合, 则在 $S^{\prime \prime} \times M^{*}$ 上,对合 $A_{0} \times I$ 与对合 $A \times \tau$ 交换, 故 $A_{0} \times I$ 诱导出 $R^{m}(\tau)=S^{m} \times M^{*} / A \times \tau$ 上的一个对合, 其不动点集法丛为

$$
(\nu \oplus m R \rightarrow F) \cup\left(\xi^{1} \rightarrow R^{m-1}(\tau)\right),
$$

其中 $\xi^{\prime}$ 为线丛, $m=0$ 时约定 $R^{-1}(\tau)$ 为空集. 由文献 [3]的 22.2 和 21.8 即知引理成立.

按文献[4],任对合 $\left(M^{*}, \tau\right)$ 也可联系一个 $n+1$ 维流形 $\Gamma\left(M^{*}\right)=S^{1} \times M^{*} / A \times \tau$ (实 际上此为 $\left.R^{1}(\tau)\right)$ 及 $\Gamma\left(M^{*}\right)$ 上的一个对合 $\tau^{(1)}, \tau^{(1)}$ 为 $A_{0} \times I$ 所诱导. 若 $\left(M^{*}, \tau\right)$ 不动 点集法丛为 $\nu \rightarrow F$, 则 $\tau^{(1)}$ 的不动点集法丛为 $(\nu \oplus R \rightarrow F) \cup\left(R \rightarrow M^{\prime \prime}\right)$. 因此, 对于对合 $\left(\Gamma\left(M^{*}\right), \tau^{(1)}\right)$, 又可联系一个对合 $\left(\Gamma\left(\Gamma\left(M^{*}\right)\right)=\Gamma^{2}\left(M^{*}\right), \tau^{(2)}\right)$, 其不动点集法丛为

$$
(\nu \oplus 2 R \rightarrow F) \cup\left(\bigcup_{0<i<2}(2-i) R \rightarrow \Gamma^{i}\left(M^{*}\right)\right) .
$$

在此约定 $\Gamma^{0}\left(M^{*}\right)-M^{*}$. 一直下去, 可联系于 $\left(M^{*}, \tau\right)$ 一个 $n+m$ 维流形 $\Gamma^{m}\left(M^{*}\right)$ 及其 上一个对合 $\tau^{(m)}$, 不动点集法丛为 $(\nu \oplus m R \rightarrow F) \cup\left(\bigcup_{0<i<m}(m-i) R \rightarrow \Gamma^{i}\left(M^{*}\right)\right)$, 对任 $m \geq$ 0.

引理 2 设 $\left(M_{1}^{*}, \tau_{1}\right),\left(M_{2}^{*}, \tau_{2}\right)$ 为两个对合, 则流形 $R^{\prime \prime}\left(\tau_{1}\right)$ 协边于流形 $R^{\prime \prime \prime}\left(\tau_{2}\right)$ 对 一切 $0 \leqslant m \leqslant k$ 当且仅当流形 $\Gamma^{\prime \prime}\left(M_{1}^{*}\right)$ 协边于流形 $\Gamma^{\prime \prime}\left(M^{\prime \prime}\right)$ 对一切 $0 \leqslant m \leqslant k$.

证 在 $k-0,1$ 时, 因任对合 $\left(M^{*}, \tau\right)$ 都有 $R^{1}(\tau)-\Gamma\left(M^{*}\right), R^{0}(\tau)-M^{*}-\Gamma^{0}$ ( $\left.M^{\prime \prime}\right)$, 故结论显然成立. 现设 $0 \leqslant m \leqslant k$ 时结论成立, 我们来证明 $0 \leqslant m \leqslant k+1$ 的情形.

记 $\nu_{i} \rightarrow F_{i}$ 为对合 $\left(M_{i}^{*}, \tau_{i}\right)$ 的不动点集法坐, $i=1,2$. 则联系于 $\left(M_{i}^{*}, \tau_{i}\right)$ 的对合 $\left(\Gamma^{k+2}\left(M_{i}^{*}\right), \tau_{i}^{(k+2)}\right)$ 的不动点集法丛为

$$
\left(\nu_{i} \oplus(k+2) R \rightarrow F_{i}\right) \cup\left(\bigcup_{0<i<k+2}(k+2-j) R \rightarrow \Gamma^{i}\left(M_{i}^{i}\right)\right) .
$$

由文献 [ 3] (p. 78, 22.1) 知, 此向量丛所对应的射影空间丛流形的协边类为 0 . 由引理 1 ,

$$
\begin{aligned}
{\left[R^{k+1}\left(\tau_{i}\right)\right]_{2} } & =\left[R P\left(\nu_{i} \oplus(k+2) R \rightarrow F_{i}\right]_{2}\right. \\
& =\sum_{i=0}^{k+1}[R P(k+1-j)]_{2} \cdot\left[\Gamma^{i}\left(M_{i}^{*}\right)\right]_{2} .
\end{aligned}
$$

因此, 若 $\left[\Gamma^{\prime \prime}\left(M_{1}^{n}\right)\right]_{2}=\left[\Gamma^{m}\left(M_{2}^{*}\right)\right]_{2}$ 对一切 $0 \leqslant m \leqslant k+1$, 则上式就给出了 $\left[R^{k+1}\left(\tau_{1}\right)\right]_{2}-$ $\left[R^{k+1}\left(\tau_{2}\right)\right]_{2}$, 连同归纳条件就有了 $\left[R^{\prime \prime}\left(\tau_{1}\right)\right]_{2}=\left[R^{\prime \prime}\left(\tau_{2}\right)\right]_{2}$ 对一切 $0 \leqslant m \leqslant k+1$. 反之, 若 $\left[R^{m}\left(\tau_{1}\right)\right]_{2}-\left[R^{m}\left(\tau_{2}\right)\right]_{2}$ 对一切 $0 \leqslant m \leqslant k+1$, 则归纳条件已给出 $\left[\Gamma^{m}\left(M_{1}^{n}\right)\right]_{2}=\left[\Gamma^{m}\left(M_{2}^{n}\right)\right]_{2}$ 对一切 $0 \leqslant m \leqslant k$, 再由上式即知也有 $\left[\Gamma^{k+1}\left(M_{1}^{*}\right)\right]_{2}=\left[\Gamma^{k+1}\left(M_{2}^{*}\right)\right]_{2}$, 故引理恒成立.

定理 1 的证明 由文献 [4](pp. 316, 命题及其证明)可推知,一个对合 $\left(M^{n}, \tau\right)$ 协边类 为 $0 \Leftrightarrow \Gamma^{m}\left(M^{*}\right)$ 的协边类为 0 对一切 $m \geqslant 0$. 现记 $M^{*}$ 为不交并 $M_{1}^{n} \cup M_{2}^{n}, \tau$ 为 $M^{*}$ 上 
的对合, $\tau \mid M_{i}^{*}=\tau_{i}, i=1,2$. 则 $\left(M_{1}^{n}, \tau_{1}\right)$ 协边于 $\left(M_{2}^{*}, \tau_{2}\right) \Leftrightarrow\left(M^{*}, \tau\right)$ 协边类为 $0 \Leftrightarrow$ $\left[\Gamma^{m}\left(M^{m}\right)\right]_{2}=0$ 对一切 $m \geqslant 0 \Leftrightarrow\left[R^{m}(\tau)\right]_{2}=0$ 对一切 $m \geqslant 0 \Leftrightarrow\left[R^{m}\left(\tau_{1}\right)\right]_{2}-\left[R^{m}\left(\tau_{2}\right)\right]_{2}$ 对一切 $m \geqslant 0$.

引理 3 设 $\left(S^{n}, \tau\right)$ 不动点集非空, 则流形 $R^{\prime \prime}(\tau)$ 的切丛的 Stiefel-Whitney 示性全 类 $W\left(R^{m}(\tau)\right)$ 必为 $1+\sum_{i=1}^{m} p^{*}\left(a_{i}\right)$. 这里 $p: R^{m}(\tau)=S^{m} \times S^{*} / A \times \tau \rightarrow R P(m)$ 为纤维丛 投射， $a_{i} \in H^{i}\left(R P(m) ; z_{2}\right)$.

证 分别以 $T R^{m}(\tau)$ 及 $T R P(m)$ 记 $R^{m}(\tau)$ 及 $R P(m)$ 的切坐. 由文献[5]分裂原 理,

$$
T R^{m}(\tau)=p^{*}(T R P(m)) \oplus \bar{T} S^{*},
$$

这里 $p^{*}(T R P(m))$ 为投射 $p$ 决定的诱导丛, $\bar{T} S^{n}$ 为 $R^{m}(\tau)$ 上沿纤维的切丛 $\bar{T} S^{*}$ 限制在 一确定纤维 $S^{n}$ 上为切丛 $T S^{n}$. 由于 $W\left(p^{*}(T R P(m))\right)=p^{*}(1+a)^{m+1}$ 已具所论形式, $a \in H^{1}\left(R P(m) ; z_{2}\right)$ 为生成元, 故只需证明 $W\left(\bar{T} S^{n}\right)=1+\sum_{i=1}^{n} V_{i}$ 也为所论形式. 以下证明 分为三步,并约定所有以下同调群都取 $z_{2}$ 系数.

(1) $\forall x_{0} \in R P(m), S^{*}=p^{-1}\left(x_{0}\right)$, 则 $p^{*}: H^{*}(R P(m)) \rightarrow H^{*}\left(R^{m}(\tau)\right)$ 及 $p^{*}: H^{*}(R P(m)$, $\left.x_{0}\right) \rightarrow H^{*}\left(R^{m}(\tau), S^{*}\right)$ 都为单射. 事实上, 因 $\left(S^{*}, \tau\right)$ 的不动点集非空, 取 $y_{0}$ 为一不动点, 则 $S^{m} \times y_{0} \leqslant S^{m} \times S^{n}$ 在 $R^{m}(\tau)=S^{m} \times S^{*} / A \times \tau$ 中自然同肧于 $R P(m)$, 故投射 $p$ 有截面.

(2) $p^{*}: H^{i}\left(R P(m), x_{0}\right) \rightarrow H^{i}\left(R^{m}(\tau), S^{*}\right), 0 \leqslant i \leqslant n$ 以及 $p^{*}: H^{i}(R P(m)) \rightarrow H^{i}\left(R^{m}(\tau)\right)$, $0 \leqslant 1 \leqslant n-1$ 都是同构. 事实上,由文献[6] Gysin 叙列知有下短正合列

$$
\begin{aligned}
& 0 \rightarrow H^{i}\left(R P(m), x_{0}\right) \stackrel{p^{*}}{\longrightarrow} H^{i}\left(R^{m}(\tau), S^{*}\right) \stackrel{\beta^{*}}{\longrightarrow} H^{i-*}\left(R P(m)_{0}\right)=0, \\
& 0 \rightarrow H^{i}(R P(m)) \stackrel{p^{*}}{\longrightarrow} H^{i}\left(R^{m}(\tau)\right) \stackrel{\beta^{*}}{\longrightarrow} H^{i-n}\left(R P(m), x_{0}\right)=0,
\end{aligned}
$$

由此可得到相应的同构.

(3) 由(2) 已知 $W\left(\bar{T} S^{n}\right)=1+\sum_{i=1}^{n} V_{i}$ 中的 $V_{i} \in p^{*} H^{i}(R P(m))$, 若 $0<i \leqslant n-1$. 故 只需证 $V_{*} \in P^{*} H^{*}(R P(m))$. 考虑以下正合交换图

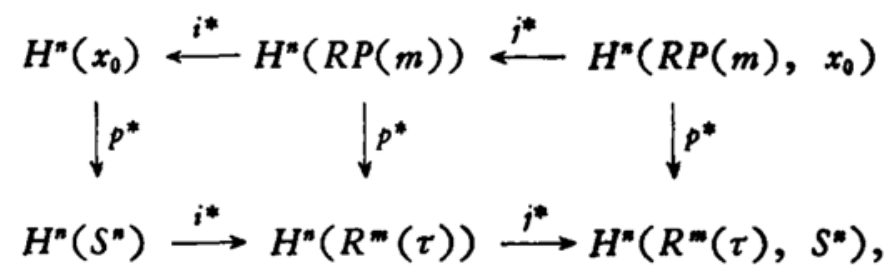

这里 $i$ 为相应的包含映射. 我们断定 $i^{*} V_{n}-0$. 这是因为 $i^{*} \bar{T} S^{*}-T S^{*}$, 而 $W\left(T S^{*}\right)-1$. 由 $i^{*} V_{n}=0$ 及正合性知有 $\alpha \in H^{n}\left(R^{m}(\tau), S^{n}\right)$ 使得 $i^{*}(\alpha)=V_{n}$, 但 $p^{*}: H^{*}\left(R P(m), x_{0}\right) \rightarrow$ $H^{n}\left(R^{m}(\tau), S^{n}\right)$ 为同构, 故再由上图表可换即知 $V_{*} \in p^{*} H^{*}(R P(m))$. 引理证完.

定理 2 的证明 (1)若 $\left(S^{n}, \tau\right)$ 为自由对合, 则由（文献 [3], 25.2) 已知对合 $\left(S^{n}, \tau\right)$ 协边类为 0 . (2) 若 $\left(S^{n}, \tau\right)$ 非自由且 $n>0$, 由引理 3 知所有 $R^{m}(\tau)$ 的 Stiefel-Whitney 示性数都为 0 , 从而 $\left[R^{m}(\tau)\right]_{2}=0$ 对一切 $m \geqslant 0$, 故定理 1 就给出了对合 $\left(S^{*}, \tau\right)$ 具 0 协边 类. 若 $n-0,\left(S^{0}, \tau\right)$ 非自由,则 $\tau$ 只能是恒同对合, 故易知此时 $\left(S^{0}, \tau\right)$ 具 0 协边类. 
由第一节, 我们已知定理 3 为定理 2 的一个直接推论, 故本文定理全部得证.

致谢: 吴振德、左再思、刘宗泽三位教授曾给予作者热情指导和豉励，在此谨致衰心感谢。

\section{參 考城}

[1]杨华起,科学通报,33(1988), 17:1356-1357.

[2] Husemoller, D., Fibre Bundles, Graduate Texts in Math., No. 2, nd edition. Springer-Verlag, New York, Heidelberg, Berlin. 1975.

[3] Conner, P. E., Diferentiable Periodic Maps, 2nd edition, Lecture Notes in Math., No. 738, SpringesVerlag, Berlin, Heidelberg, New York, 1979.

[4] Kosniowski, C. and Stong, R. E., Topology, 17(1978), 4-A: 309-316.

[5] Borel, A. and Hirzebruch, F., Am. J. Math., 80(1958), 482.

[6] Whitehend, G. W., Elements of Homotopy Theory, Springer-Verlag, New York, Heidelberg, Berlin, 1978, 356. 